\title{
Medidas preventivas para diminuição no risco de pneumonia associada à ventilação mecânica
}

\author{
Preventive measures to decrease the risk of pneumonia associated with mechanical ventilation
}

\author{
Bruno César Fernandes $^{1^{*}} \bullet$, Alan Márcio de Brito Araújo ${ }^{\oplus}$, Nédson Lechner da Silva ${ }^{1} \bullet$, Lígia \\ Harumi Vilela Bartnick Tanaka ${ }^{2}$, Catherine Alexia Yoshikawa ${ }^{2}$, , Flávio Henrique Souza de \\ Araújo $^{3}$
}

\begin{abstract}
${ }^{1}$ Hospital Universitário da Universidade Federal Grande Dourados, Ebserh, Dourados, Mato Grosso do Sul, Brasil. ${ }^{2}$ Faculdade de Ciências da Saúde, Universidade Federal da Grande Dourados, Dourados, Mato Grosso do Sul, Brasil. ${ }^{3}$ Programa de Pós-Graduação em Ciências da Saúde, Faculdade de Ciências da Saúde, Universidade Federal da Grande Dourados, Dourados, Mato Grosso do Sul, Brasil. *Autor para correspondência. E-mail: brunoanaisafernandes@gmail.com.
\end{abstract}

\begin{abstract}
Resumo: Introdução: A ventilação mecânica invasiva expõe os pacientes ao risco de adquirir pneumonia associada à ventilação mecânica, o que provoca altos índices de internação prolongada, aumento dos custos hospitalares e consequente agravo no quadro clínico, elevando a mortalidade desses pacientes. Tais aspectos tornam a pneumonia associada à ventilação mecânica a segunda principal infecção relacionada à assistência à saúde em unidade de terapia intensiva. Revisão: Por meio da metodologia de revisão bibliográfica, esse estudo teve como objetivo identificar, conhecer e reforçar as medidas preventivas realizadas pelos cuidados de enfermagem, capazes de diminuir a incidência de pneumonia associada à ventilação mecânica em unidade de terapia intensiva. Discussão: Foram selecionadas 22 referências, sendo 19 artigos científicos, um livro e duas publicações de organizações de saúde, todos em português. Considerações finais: Com base na literatura consultada, concluiu-se que a adesão às medidas preventivas é um poderoso instrumento para definir estratégias que aumentem a qualidade da assistência à saúde em pacientes de unidade de terapia intensiva em ventilação mecânica invasiva. Sugere-se que medidas educativas direcionadas à implementação de bundles para a prevenção de pneumonia associada à ventilação mecânica sejam desenvolvidas para toda a equipe de saúde que atua em unidade de terapia intensiva, em especial para a equipe de enfermagem, cujos profissionais são os que mais estão relacionados com este tipo de cuidado.
\end{abstract}

Palavras-chave: Pneumonia associada à ventilação mecânica, respiração artificial, cuidados de enfermagem.

\begin{abstract}
Introduction: Invasive mechanical ventilation exposes patients to the risk of acquiring pneumonia associated with mechanical ventilation, which causes high rates of prolonged hospitalization, increased hospital costs and consequent worsening of the clinical picture, increasing the mortality of these patients. Such aspects make pneumonia associated with mechanical ventilation the second main infection related to health care in an intensive care unit. Review: Through the literature review methodology, this study aimed to identify, know and reinforce preventive measures performed by nursing care, capable of reducing the incidence of pneumonia associated with mechanical ventilation in an intensive care unit. Discussion: 22 references were selected, including 19 scientific articles, a book and two publications by health organizations, all in Portuguese. Final considerations: Based on the consulted literature, it was concluded that adherence to preventive measures is a powerful instrument to define strategies that increase the quality of health care in patients in intensive care units in invasive mechanical ventilation. It is suggested that educational measures aimed at the implementation of bundles for the prevention of pneumonia associated with mechanical ventilation be developed for the entire health team that works in an intensive care unit, especially for the nursing team, whose professionals are the ones who most are related to this type of care.
\end{abstract}

Keywords: Pneumonia associated with mechanical ventilation, artificial respiration, nursing care.

\section{Introdução}

A Pneumonia associada a ventilação mecânica invasiva (PAVMI) é uma infecção relacionada à assistência à saúde (IRAS) de grande importância para o sistema público de saúde do Brasil, uma vez que, a PAVMI está inserida no rol das principais IRAS adquiridas no âmbito da Unidade de Terapia Intensiva (UTI) representando importante causa de óbitos, em igual escala quando comparada às causadas por doenças 
transmissíveis, cardiovasculares, respiratórias e neoplásicas, tornando, ainda mais, evidente o grande impacto que a sua ocorrência representa ao sistema público de saúde brasileiro. (Bork et al., 2015).

Entre os principais fatores de risco no desenvolvimento da pneumonia nosocomial tem-se: implementação terapêutica inadequada de antibióticos; pacientes idosos; presença e gravidade de doenças de base; desnutrição; uso de cânulas e cateteres nasogástricos; utilização prolongada de dispositivos terapêuticos invasivos, tais como, cateter semi-implantado para infusão de medicamentos e cateteres vesicais; inserção de tubo orotraqueal para suporte ventilatório; traqueostomia; condição clínica do paciente; internação por um período de cinco ou mais dias; pacientes com alteração no nível de consciência ou em uso de medicação imunossupressora; antiácidos e bloqueadores de receptores $\mathrm{H} 2$; deslocamento do paciente por ambientes intra-hospitalar (por ex. para realização de uma tomografia); permanência na posição supina e ventilação mecânica invasiva prolongada (SBPT, 2007; Mota et al., 2017).

No caso da ventilação mecânica invasiva (VMI), sua realização é de suma importância para reversão e recuperação de pacientes acometidos com patologias graves relacionadas ao aparelho respiratório, sendo a intubação traqueal um grande alicerce nessa terapêutica (AMIB \& SBPT, 2013).

No entanto, devido às alterações provocadas por esse procedimento, que utiliza uma cânula endotraqueal ou traqueostomia, os mecanismos de defesa naturais são rompidos. Como consequência, deflagam-se diversos eventos, indesejáveis, no paciente como a instabilidade hemodinâmica, predisposição a maior frequência de infecções respiratórias e lesões físicas, comprometendo de forma significativa a recuperação. Isso provoca, então, altos índices de internação prolongada, aumento dos custos hospitalares e consequente agravo no quadro clínico e, assim, aumento da mortalidade (Nascimento et al., 2017).

A PAVMI é uma das principais complicações provocadas pela respiração artificial, e é um processo infeccioso do parênquima pulmonar com mais de $80 \%$ dos casos provocados por bactérias. Ela ocorre cerca de 48 horas ou mais após a admissão hospitalar, e quando a doença não estava incubada no momento da hospitalização (Cheever \& Hinkle, 2016).

Diante da importância desse agravo à saúde, o presente estudo buscou identificar quais os impactos da adoção de medidas preventivas, relacionadas à assistência de enfermagem, na diminuição da incidência da PAVMI em UTI. Além disso, visou-se conhecer e reforçar as medidas comprovadamente capazes de gerar essa diminuição.

\section{Revisão}

Trata-se de uma pesquisa bibliográfica, no intuito de reunir informações sobre, medidas preventivas para PAVMI, por meio de artigos científicos encontrados na Biblioteca Virtual em Saúde. A pesquisa, nas bases de dados consultadas, foi realizada utilizando os seguintes descritores: pneumonia associada a ventilação mecânica; respiração artificial; cuidados de enfermagem.

Os seguintes critérios de inclusão foram adotados: data de publicação entre 2000 e 2020; no idioma português (já que buscou-se estudar a realidade brasileira); disponíveis na íntegra e online. Além dos artigos, também, foram consultadas publicações oficiais de organizações de saúde (como a Agência Nacional de Vigilância Sanitária, Sociedade Brasileira de Pneumologia e Tisiologia e Associação de Medicina Intensiva Brasileira), que foram escolhidos por possuírem informações relevantes ao tema. Posteriormente foram selecionadas 22 referências, sendo 19 artigos científicos, um livro e duas publicações de organizações de saúde, todos em português.

\section{Discussão}

Tendo em vista a importância e a complexidade da PAVMI, torna-se premente a realização de intervenções que causam impactos significativos na prevenção destas ocorrências. Neste sentido, tem sido adotada a estratégia da criação de bundles (pacotes) de cuidados, os quais reúnem um pequeno grupo de intervenções que, quando utilizadas em conjunto, mostram-se mais eficazes para assistência em saúde do que quando individualmente (Almeida et al., 2015; ANVISA, 2017).

Os bundles, ou pacote de medidas, englobam estratégias de cuidados implementadas no cuidado do paciente com riscos de desenvolver IRAS, neste caso a PAVMI. Os cuidados contidos nos bundles são avaliados pela Comissão de Controle de Infecção Hospitalar quanto a sua pertinência, aplicabilidade e considerando o contexto onde se desenvolve, sendo atribuídos o protagonismo de sua execução à equipe multidisciplinar. Além disso, não exigem tecnologias complexas, nem aumento na carga de trabalho dos 
profissionais, e tampouco custos adicionais para a instituição, o que permite sua aplicação em qualquer UTI (Almeida et al., 2015).

Entre as medidas indicadas como preventivas para PAVMI, tem-se a manutenção da cabeceira elevada entre 30 e $45^{\circ}$, algo relativamente simples e sem custos adicionais. Ela é considerada uma das principais recomendações para evitar a ocorrência de broncoaspiração, que causa a PAVMI, além de contribuir para uma melhoria no volume corrente e ventilatório, diminuindo, também, os casos de atelectasia (Almeida et al., 2015).

Outra recomendação, feita pela Agência Nacional de Vigilância Sanitária (ANVISA), é a aspiração da secreção subglótica rotineiramente, já que, em pacientes sob VMI, a presença do tubo endotraqueal facilita a colonização bacteriana da árvore traqueobrônquica e a aspiração da secreção contaminada, por diminuir o reflexo de tosse (ANVISA, 2017). Por isso, é recomendado o uso de tubos traqueais, visto que permitem a aspiração intermitente da secreção acumulada acima do balonete (cuff), com pressões altas, ou contínuas. Essa medida visa mitigar a ocorrência PAVMI por microaspirações de secreção contaminada que tende a acumular na região medial da traqueia, acima do balonete (Barbas \& Couto, 2012; Souza \& Santana, 2012).

Vale destacar, também, os cuidados com o equipamento de ventilação, devendo-se verificar a posição do filtro umidificador conectado ao tubo orotraqueal, o qual deve estar elevado acima da região da traqueia, além de verificar a ausência de líquidos no circuito das traqueias e no filtro (Sachetti et al., 2014).

Um fator predisponente ao desenvolvimento de PAVMI é a administração contínua de sedativos, tendo em vista que condiciona maior duração da VMI, maior permanência na UTI e no hospital. Portanto, uma ótima medida de prevenção é a interrupção diária da sedação, assim como a avaliação da possibilidade de extubação dos pacientes. Ambas são altamente recomendadas para prevenção da PAVMI, haja vista que pacientes despertos podem contribuir para o desmame ventilatório (Almeida et al., 2015).

Outra medida indispensável é a cultura de higienização das mãos pelos profissionais de saúde, sendo recomendado que a temática seja abordada em intervenções educativas para sensibilizar quanto a sua importância na prevenção e controle das IRAS ressaltando que a eficácia da higienização das mãos é reflexo da periodicidade e técnica corretas empregadas no processo de higienização das mãos, compreendendo inclusive, o produto utilizado, podendo ser agua e sabão ou produtos com agentes antissépticos como o álcool, por exemplo (ANVISA, 2017).

Ademais é fortemente recomendado a descontinuação da utilização de adornos pelos profissionais de saúde durante suas atividades laborais, pois pulseiras, correntes, anéis, relógios e brincos são considerados locais de acumulo e difícil remoção de microrganismos, podendo ser um veículo de disseminação de IRAS (ANVISA, 2017).

Além disso, uma estratégia adicional para a prevenção de PAVMI é a prática, frequente, da higienização adequada da cavidade oral de pacientes em VMI, pois estes, apresentam diminuição em sua produção de saliva e impossibilidade de mastigação. Tais fatos favorecem o aparecimento de biofilme dental, um importante reservatório para microrganismos. Outrossim, como a PAVMI ocorre devido à aspiração do conteúdo da orofaringe, segue-se a lógica de buscar a erradicação da colonização bacteriana desta região (Almeida et al., 2015). Por isso, a ANVISA (2017) indica o uso de clorexidina 0,12\% para higiene oral. Isto, quando aplicado conjuntamente a outras medidas, parece ter um efeito positivo para redução da PAVMI.

Paralelamente, a realização de profilaxia de trombose venosa profunda (TVP) é outra medida que, embora não esteja associada de maneira direta à prevenção da PAVMI, possui impacto na diminuição da mortalidade hospitalar e do tempo de internação. Não só isso, as complicações relacionadas com a TVP retardam a extubação do paciente, aumentando, então, o tempo de VMI e predispondo o paciente à PAVMI (Almeida et al., 2015).

Também é indicada a profilaxia de úlcera gástrica, pois a VMI é um fator de risco para sua formação, e alguns autores recomendam a adoção de medidas que evitem o aumento do pH estomacal. A úlcera de estresse, quando diagnosticada, pode provocar hemorragia digestiva, o que piora as condições clínicas do paciente e retarda sua saída da VMI, aumentando, assim, os riscos de PAVMI (Almeida et al., 2015).

Existem estudos desenvolvidos especificamente sobre medidas preventivas da PAVMI, como o elaborado por Almeida et al. (2015), que descreve o seguinte bundle de prevenção de PAVMI: elevação da cabeceira da cama em $30-45^{\circ}$, realização de profilaxia de trombose venosa profunda (TVP), profilaxia de úlcera péptica, interrupção da sedação, higiene oral e mensuração da pressão do cuff (para assegurar a vedação da traqueia e impedir microaspirações de secreções subglóticas para o trato respiratório inferior).

Em estudo realizado por Rodrigues et al. (2016) em um Hospital Público de Ensino, em Fortaleza (CE), o bundle implementado consistia em: Identificação do RASS (escala de agitação e sedação de Richmond), para avaliação do nível de sedação; interrupção diária da sedação; elevação da cabeceira entre 30 a $45^{\circ}$; nutrição 
enteral ( administração de dieta por sonda é fator predisponente para a PAVMI, pelo risco de aspiração do conteúdo gástrico); critérios no uso de bloqueio neuromuscular, (pode induzir uma disfunção neuromuscular, o que dificulta o desmame ventilatório); manutenção da pressão adequada do cuff; e higiene oral com clorexidina aquosa a $0,12 \%$.

Outro bundle, descrito por Sachetti et al. (2014), inclui: posição da cabeceira entre $30^{\circ}$ e $45^{\circ}$; posição do filtro umidificador conectado ao tubo orotraqueal acima da região da traqueia; verificação da ausência de líquidos no circuito das traqueias e no filtro umidificador; higiene oral no mínimo uma vez por turno, ou seja, três vezes ao dia, com uso de clorexidina; verificação da pressão do cuff do tubo orotraqueal diariamente (deve estar entre 20 e $30 \mathrm{cmH}_{2} \mathrm{O}$ ); e, por fim, realização de fisioterapia três vezes ao dia.

Barbas e Couto (2012) listam as seguintes medidas, em que se observa concordância com algumas já citadas: elevação da cabeceira do leito de 30 a $45^{\circ}$; higiene oral com clorexidina; realização do despertar diário do paciente; protocolos efetivos de desmame da ventilação mecânica; higiene das mãos dos profissionais de saúde; utilização de tubos traqueais com antissépticos; utilização de tubos com possibilidade de aspiração de secreção suprabalonete; e aumento da utilização de ventilação não invasiva.

Outro grupo de pesquisadores (Bork et al., 2015), relacionam as seguintes medidas: cabeceira elevada entre $30^{\circ}$ e $45^{\circ}$; interrupção da sedação; higiene oral com clorexidina; pressão do cuff orotraqueal entre 20 e $25 \mathrm{mmH}_{2} \mathrm{O}$; filtro de nebulizador dentro do prazo de validade e circuito sem a presença de condensados.

No estudo realizado em um hospital público de Santa Catarina, por Silva et al. (2012), o bundle implementado incluía: cabeceira elevada $30-45^{\circ}$; aspiração endotraqueal; pressão do cuff entre 20-30 $\mathrm{cmH2O}$ e higiene oral com clorexidina $0,12 \%$. Por serem de mais fácil aplicação e adesão, essas quatro medidas foram recomendadas, posteriormente, como as principais pelos autores (Silva et al., 2014). Já o estudo de Perugini et al. (2015), direcionado para pacientes infantis, alerta que a elevação da cabeceira do leito para recém-nascidos deve ser entre 15 a $30^{\circ}$, sendo de 30 a $45^{\circ}$ apenas para crianças maiores.

Em relação à adesão a medidas preventivas, uma série de estudos (Pombo et al., 2010; Silva et al., 2011; Silva et al., 2012; Gonçalves et al., 2012; Silva et al., 2014; Rodrigues et al., 2016) revelam uma baixa adesão às medidas de prevenção, em especial a interrupção da sedação e a higiene oral, que é, segundo Almeida et al. (2015), um cuidado imprescindível em pacientes submetidos à VMI. De acordo com outros estudos (Silva et al., 2011; Silva et al., 2012; Silva et al., 2014; Rodrigues et al., 2016; Silva et al., 2017), essa baixa adesão se dá por falta de conhecimento e preparo dos profissionais.

De acordo com Nascimento et al. (2017), um cuidado interdisciplinar para a prevenção pode oferecer inúmeras vantagens ao paciente crítico, como a implementação de bundles, que substituem as medidas isoladas de prevenção, fato esse baseado em evidências científicas. Ademais, são essenciais ações educativas permanentes e a valorização da Sistematização da Assistência de Enfermagem na UTI frente às prescrições de enfermagem voltadas à profilaxia da pneumonia. Inclusive, conforme explicam Gonçalves et al. (2012), a responsabilidade da equipe de enfermagem no controle e prevenção da PAVMI é fundamental, pois são estes profissionais que realizam a maioria dos cuidados relativos ao uso de VMI na UTI.

Outros autores afirmam que quando a equipe de saúde aplica todas as medidas preventivas ao paciente sob ventilação mecânica há redução do risco de se adquirir infecção no trato respiratório (Pombo et al., 2010; Silva et al., 2011; Gonçalves et al., 2012; Sachetti et al., 2014; Silva et al., 2014; Rodrigues et al., 2016; Silva et al., 2017; Mansano et al., 2017) e, segundo Silva et al. (2014), Bork et al. (2015) e Nascimento et al. (2017), as ações educativas apresentam impacto positivo na adesão às práticas de prevenção da PAVMI.

\section{Considerações finais}

Por meio dessa pesquisa foi possível constatar que pacientes em uso de tubo endotraqueal e suporte ventilatório invasivo, ou seja, sob VMI, caso não sejam assistidos com cautela e os equipamentos manuseados da forma recomendada, poderão estar em risco do desenvolvimento de PAVMI. Isso deve-se ao fato de, por se tratar de pacientes vulneráveis e hemodinamicamente instáveis, é necessária a utilização de medidas preventivas para aumentar a segurança e a qualidade do atendimento na UTI.

Os resultados de alguns dos artigos pesquisados demonstraram certa fragilidade em relação aos cuidados assistenciais em saúde na UTI, levando à exposição dos pacientes a situações que predispõem o desenvolvimento de PAVMI. Portanto, a sensibilização da equipe quanto a importância dos bundles e a implantação de medidas de prevenção de PAVMI é necessária e urgente, pois impacta diretamente na segurança e qualidade de assistência ao paciente. Além disso, reduz custos e poupa o paciente e sua família de todas as implicações clínicas que uma infecção do trato respiratório pode causar. 
De acordo com a maioria dos estudos, há um grupo de ações que se destacam como medidas imprescindíveis na prevenção de PAVMI. São elas: elevação da cabeceira em $30-45^{\circ}$; interrupção da sedação diariamente; aspiração endotraqueal; pressão do cuff entre 20-30 cmH2O; higiene oral com clorexidina 0,12\%; higienização das mãos; posição e manutenção do equipamento de ventilação. A adesão a essas medidas preventivas é um poderoso instrumento para definir estratégias que melhorem a qualidade da assistência à saúde em pacientes de UTI em VMI.

Diante do exposto, sugere-se que medidas educativas direcionadas à implementação de bundles para a prevenção de PAVMI sejam desenvolvidas para toda a equipe de saúde que atua em UTI. Sobretudo, deve-se ter um enfoque especial para a equipe de enfermagem, cujos profissionais são os mais relacionados a este tipo de cuidados aos pacientes em VMI.

\section{Referências}

Agência Nacional de Vigilância Sanitária [ANVISA]. 2017. Medidas de prevenção de infecção relacionada à assistência à saúde. Brasília, DF: ANVISA.

Almeida, K .M. V., Barros, O. M .C., Santos, G. J. C., Valença, M. P., Cavalcanti, A. T. A. \& Ferreira, K. O. Adesão às medidas de prevenção para pneumonia associada à ventilação mecânica. Revista Enfermagem UFSM, 5(2), 247-256. 2015

Associação De Medicina Intensiva Brasileira [AMIB] \& Sociedade Brasileira de Pneumologia e Tisiologia. Diretrizes brasileiras de ventilação mecânica [SBPT]. 2013. I Fórum de diretrizes em ventilação mecânica AMIB e SBPT. São Paulo, SP: AMIB, SBPT.

Barbas, C .S. V. \& Couto, L. P. 2012. Tubos endotraqueais com aspiração suprabalonete diminuem a taxa de pneumonia associada à ventilação mecânica e são custo-efetivos?. Revista Brasileira de Terapia Intensiva, 24(4), 320-321.

Bork, L. C. A., Gaspar, M. D .R. \& Reche, P. 2015. Adesão às medidas preventivas de pneumonia associada à ventilação mecânica. Rev Epidemiol Control Infect, 5(1), 12-16.

Gonçalves, F. A. F., Brasil, V. V., Ribeiro, L. C. M. \& Tipple, A. F. V. 2012. Ações de enfermagem na profilaxia da pneumonia associada à ventilação mecânica. Acta paul. Enfermagem, 25(spe1), 101-107.

Gonçalves, F. A. F., Brasil, V. V., Minamisava, R., Caixeta, C. R., Oliveira, L. M. A. C. \& Cordeiro, J. A. B. L. 2012.Eficácia de estratégias educativas para ações preventivas da pneumonia associada à ventilação mecânica. Escola Anna Nery, 16(4), 802-808.

Mansano, F. P. N., Belei, R. A., Vinci, L. A. S., Melo, B. L. D., Cardoso, L. T. Q., Garcia, J. C. P., Carrilho, C. M. D. M., Ezaias, G. M. \& Grion, C. M. C. 2017. Impacto de ação educativa na manutenção do decúbito elevado como medida preventiva de pneumonia associada à ventilação mecânica em Unidade de Terapia Intensiva. ABCS Health Sciences, 42(1), 21-26.

Mota, E. C., Oliveira, S. P., Silveira, B. R. M., Silva, P. L. N. \& Oliveira, A. C. 2017. Incidência da pneumonia associada à ventilação mecânica em unidade de terapia intensiva. Medicina, 50(1), 39-46.

Nascimento, T .B. P., Ramos, C. H., Silva, T. S. \& Torres, V. P. S. 2017. Efetividade das medidas de prevenção e controle de pneumonia associada à ventilação mecânica na UTI. Perspectivas online: biológicas e saúde, 25(7), $1-24$.

Cheever, K. H. \& Hinkle, J. L. 2016. Brunner \& Suddarth - tratado de enfermagem médico cirúrgica (13a ed.). Rio de Janeiro, RJ: Guanabara Koogan.

Souza, C .R. \& Santana, V. T. S. 2012. Impacto da aspiração supra-cuff na prevenção da pneumonia associada à ventilação mecânica. Revista Brasileira de Terapia Intensiva, 24(4), 401-406.

Perugini, M. R. E., Perugini, V. H., Figueira, F. D., Fontana, L. M. S., Diniz, J. J., dos Santos, D. L., ... \& Capobiango, J. D. (2015). Impacto de um bundle nas taxas de pneumonia associada à ventilação mecânica (PAV) em uma unidade de terapia intensiva pediátrica em Londrina-PR. Semina: Ciências Biológicas e da Saúde, 36(1Supl), 259-266.

Pombo, C. M. N., Almeida, P. C. \& Rodrigues, J. L.N. 2010. Conhecimento dos profissionais de saúde na Unidade de Terapia Intensiva sobre prevenção de pneumonia associada à ventilação mecânica. Ciência Saúde Coletiva, 15(Suppl 1), 1061-1072.

Rodrigues, N. A., Fragoso, L. V. C., Beserra, F. M. \& Ramos, I. C. Impactos e fatores determinantes no bundle de pneumonia associada à ventilação mecânica. Revista Brasileira de Enfermagem, 69(6),1108-1114. 2016. 
Sachetti, A., Rech, V., Dias, A. S., Fontana, C., Barbosa, G. L. \& Schlichting, D. 2014. Adesão às medidas de um bundle para prevenção de pneumonia associada à ventilação mecânica. Revista Brasileira de Terapia Intensiva, 26(4), 355-359.

Silva, L. T. R., Laus, A. M., Canini, S. R. M. S. \& Hayashida, M. 2011. Avaliação das medidas de prevenção e controle de pneumonia associada à ventilação mecânica. Revista Latino-Americana de Enfermagem, 19(6),1329-1336.

Silva, S. G., Nascimento, E. R. P. \& Salles, R. K. 2012. Bundle de prevenção da pneumonia associada à ventilação mecânica: uma construção coletiva. Texto \& Contexto Enfermagem, 21(4), 837-844.

Silva, S. G., Nascimento, E. R. P. \& Salles, R. K. 2014. Pneumonia associada à ventilação mecânica: discursos de profissionais acerca da prevenção. Esc. Anna Nery,18(2), 290-295.

Silva, S. G., Salles, R. K., Nascimento, E. R. P., Bertoncello, K. C. G. \& Cavalcanti, C. D. K. 2014. Avaliação de um bundle de prevenção da pneumonia associada à ventilação mecânica em unidade de terapia intensiva. Texto \& Contexto Enfermagem, 23(3), 744-750.

Silva, T. G., Souza, G. N., Souza, S. S., Bitencourt, J. V. V., Madureira, V. F. \& Luzardo, A. R. 2017. Incidência de pneumonia associada à ventilação mecânica em uma unidade de terapia intensiva. Revista de Pesquisa: Cuidado é Fundamental, 9(4),1121-1125. 2017.

Sociedade Brasileira De Pneumologia E Tisiologia [SBPT]. 2007. Diretrizes brasileiras para tratamento das pneumonias adquiridas no hospital e das associadas à ventilação mecânica. Jornal Brasileiro de Pneumologia, 33(Supl 1), S1-S30.

\section{Minicurrículo}

Bruno César Fernandes. Enfermeiro Assistêncial da Empresa Brasileira de Serviços Hospirtalares (EBERH/HU-UFGD), mestrando do Programa de Pós-Graduação Stricto Sensu Mestrado Profissional Ensino em Saúde da Universidade Estadual do Mato Grosso do Sul (PPGES-UEMS) polo Dourados/MS. Pós-graduado em Unidade de Terapia Intensiva, Unidade de Terapia Intensiva Neonatal e Pediátrica (Ingá) e, em Saúde da Família.

Alan Márcio de Brito Araújo. Enfermeiro Assistêncial da Empresa Brasileira de Serviços Hospirtalares (EBERH/HU-UFGD). Especialização em Saúde da Família, Terapia Intensiva Adulto, e, Residência Multiprofissional em Neurologia.

Nédson Lechner da Silva. Possui graduação em Negócios Imobiliários e graduação em Enfermagem. Atualmente é Funcionário Público da EBSERH HU/UFGD, ocupando o cargo de Enfermeiro Assistencial, lotado na UTI Adulto. Especialização em Práticas Avançadas de Enfermagem na Atenção Primária.

Lígia Harumi Vilela Bartnick Tanaka. Graduanda do curso de Medicina pela Universidade Federal da Grande Dourados.

Catherine Alexia Yoshikawa. Graduanda do curso de Medicina pela Universidade Federal da Grande Dourados. Em 2019, atuou como monitora voluntária da disciplina de Tecidos I e bolsista da disciplina de Anatomia Humana. Atualmente, é Bolsista de Iniciação Tecnológica do CNPq, coordenadora de pesquisa da Liga Acadêmica de Diabetes e Hipertensão Arterial Sistêmica (LADHAS) e membro da Liga Acadêmica de Anatomia Clínica e Cirúrgica (LACC).

Flávio Henrique Souza de Araújo. Graduado em Ciências Biológicas e Educação Física Bacharelado. Atualmente é Doutorando no Programa de Pós-graduação em Ciências da Saúde na Faculdade de Ciências da Saúde na Universidade Federal da Grande Dourados (FCS/UFGD). 
Como citar: Fernandes, B.C., Araújo, A.M.B., Silva, N.L., Tanaka, L.H.V.B., Yoshikawa, C.A., \& Araújo, F.H.S. 2021. Medidas preventivas para diminuição no risco de pneumonia associada à ventilação mecânica, 6, 133. DOI: https://dx.doi.org/10.31533/pubsaude6.a133

Recebido: 12 fev. 2021.

Revisado e aceito: 1 mar. 2021.

Conflito de interesse: os autores declaram, em relação aos produtos e companhias descritos nesse artigo, não ter interesses associativos, comerciais, de propriedade ou financeiros que representem conflito de interesse.

Licenciamento: Este artigo é publicado na modalidade Acesso Aberto sob a licença Creative Commons Atribuição 4.0 (CC-BY 4.0). 